\title{
Consumo de mídias sexualmente explícitas e sexo anal desprotegido em homens que fazem sexo com homens
}

\author{
Consumption of sexually explicit media and unprotected anal sex \\ in men who have sex with men
}

\author{
Anderson de Araújo Martins (https://orcid.org/0000-0003-4964-3595) ${ }^{1}$ \\ Artur Acelino Francisco Luz Nunes Queiroz (https://orcid.org/0000-0002-6350-1908) ${ }^{2}$ \\ Oleci Pereira Frota (https://orcid.org/0000-0003-3586-1313) ${ }^{1}$ \\ Telma Maria Evangelista de Araújo (https://orcid.org/0000-0001-5628-9577) ${ }^{3}$ \\ Isabel Amélia Costa Mendes (https://orcid.org/0000-0002-0704-4319) ${ }^{2}$ \\ Inês Fronteira (https://orcid.org/0000-0003-1406-4585) ${ }^{4}$ \\ Álvaro Francisco Lopes de Sousa (https://orcid.org/0000-0003-2710-2122) ${ }^{4}$
}

${ }^{1}$ Instituto Integrado de Saúde, Universidade Federal de Mato Grosso do Sul. Cidade Universitária, s/n Universitário -900. 79070-900 Campo Grande MS Brasil.

sousa.alvaromd@gmail.com ${ }^{2}$ Escola de Enfermagem de Ribeirão Preto, Universidade de São Paulo. São SP Brasil.

${ }^{3}$ Departamento de Enfermagem, Universidade Federal do Piauí. Teresina PI Brasil.

${ }^{4}$ Global Health and

Tropical Medicine, Instituto de Higiene e Medicina

Tropical, Universidade Nova de Lisboa. Lisboa Portugal.
Abstract This study aimed to evaluate the influence of bareback sexually explicit media (SEM) consumption on anal sex without a condom by men who have sex with men (MSM). To this end, a page was created on the Facebook ${ }^{\circledR}$ social network with a link that directed interested parties to a questionnaire. Cisgender men, aged 18 years and over, who had sex with other men in the last 12 months, were included. Data were collected in 2017 and analyzed using univariate and bivariate inferential statistics and multivariate logistic regression. A total of 2,248 MSM participated in the research, with a mean age of 24.4 years and $a$ mean number of 3.9 partners in the last 30 days. Having multiple sexual partners (ORa: 9.4; 95\% CI 3.9-22.4), preferring movies with bareback scenes (ORa: 2.6; 95\% CI 1.5-4.6), considering this practice a fetish and realizing it (ORa: 3.52; 95\% CI 2.3-5.4), having casual partnerships (ORa: 1.8; 95\% CI 1.5-1.9) and being aware of the partner's negative serological status for HIV (ORa: 1.4; 95\% CI 1.1-2.3) were factors that increased the likelihood of engaging in anal sex without a condom. Thus, we found an association between the consumption of bareback SEM and sex without a condom among MSM.

Key words Audiovisual media, Sexual behavior, Unprotected sex, Condoms, Sexual and gender Minorities
Resumo O objetivo deste estudo foi avaliar a influência do consumo de mídia sexualmente explícita (MSE) de modalidade bareback na prática de sexo anal sem preservativo por homens que fazem sexo com homens (HSH). Para tanto, foi criada uma página na rede social Facebook ${ }^{\circledR}$ com um link que direcionava os interessados para um questionário. Foram incluídos homens cisgênero, com 18 anos ou mais e que praticaram sexo com outro(s) homem(ns) nos últimos 12 meses. Os dados foram coletados em 2017 e analisados por meio de estatística inferencial (uni)bivariada $e$ regressão logística multivariada. Participaram da pesquisa 2.248 HSH, com média de idade de 24,4 anos e média de 3,9 parceiros nos últimos 30 dias. Possuir múltiplos parceiros sexuais (ORa:9,4; IC95\% 3,9-22,4), preferir filmes com cenas bareback (ORa:2,6; IC95\% 1,5-4,6), julgar essa prática um fetiche e realizá-lo (ORa:3,52; IC95\% 2,3-5,4), ter parceria casual (ORa:1,8; IC95\% 1,5-1,9) e ciência do status sorológico negativo do parceiro para o HIV (ORa:1,4; IC95\% 1,1-2,3) foram fatores que aumentaram as chances de envolvimento em sexo anal sem preservativo. Dessa forma, verificamos associação entre o consumo de MSE bareback e a prática de sexo sem preservativo entre HSH.

Palavras-chave Midia audiovisual, Comportamento sexual, Sexo sem proteção, Preservativos, Minorias sexuais e de gênero 


\section{Introdução}

As mídias sexualmente explícitas (MSE) compreendem qualquer tipo de material com descrição de órgãos genitais ou atos sexuais explícitos de qualquer natureza, capazes de provocar ou modificar sentimentos ou pensamentos sexuais do espectador ${ }^{1}$. O avanço tecnológico possibilitou a expansão e facilitou o acesso às MSE, principalmente entre indivíduos do sexo masculino, incluindo os homens que fazem sexo com homens (HSH), sendo seu consumo amplamente aceito entre estes ${ }^{2}$.

O efeito do consumo das MSE na saúde sexual dos HSH é motivo de controvérsias na literatura. Alguns estudos identificam influências positivas do consumo de MSE no desenvolvimento sexual e nas práticas sexuais dos $\mathrm{HSH}$ pois muitos adolescentes e jovens utilizam essas mídias como fonte de informação para aprenderem sobre identidade sexual, como praticar sexo com outros homens, compreender seus desejos ${ }^{3,4}$, melhorar a autoeficácia do uso do preservativo e aumentar o interesse em sexo seguro ${ }^{5}$. No entanto, outros relatam influências negativas no comportamento sexual, como a aceitabilidade da prática de sexo sem preservativo como algo comum ${ }^{6}$. As diferenças encontradas entre estudos podem estar associadas a recentes mudanças nas MSE no que concerne ao uso do preservativo nas cenas, embora não haja consenso sobre essa associação.

Alarmados pela epidemia de Aids, os estúdios pornográficos gays produziram quase exclusivamente filmes com preservativo até o final da década de 1990 e início dos anos 2000. A partir da segunda metade da década de 2000 , entretanto, a indústria homoerótica presenciou uma crescente tendência na produção de filmes tipo bareba$c k$, termo em inglês que se refere a um estilo de montaria a cavalo em que o "vaqueiro não usa sela". A expressão tem sido amplamente utilizada por HSH para designar o sexo no qual intencionalmente se abre mão do uso de preservativo ${ }^{7-9}$.

Esses filmes frequentemente mostram troca de sêmen entre os atores, prática que hoje se tornou quase universal entre os produtores de MSE gay ${ }^{10,11}$. Nos Estados Unidos, um dos maiores produtores de MSE no mundo, apenas dois dos grandes estúdios (GayHoopla e GayRoom) continuam usando preservativos, sendo a grande maioria das novas cenas sexuais entre gays praticadas na modalidade bareback ${ }^{12}$.

Uma das razões para essa expansão das MSE sem preservativo é a necessidade em atender o aumento na busca dos espectadores por vídeos que retratam essa prática, os quais relatam que elas representam o sexo mais natural, ou seja, mais próximo do real e que podem apresentar diferentes significados dependendo do cenário ou contexto apresentado, das características dos atores envolvidos e das relações de poder observadas na cena ${ }^{11}$. Outros fatores que favoreceram o crescimento desse tipo de MSE foram os avanços na terapia antirretroviral (TARV), o surgimento da PrEP com a combinação de Emtricitabina com Tenofovir como profilaxia pré-exposição para o HIV e a testagem sorológica como formas de garantir a produção de filmes bareback e assegurar a saúde sexual dos atores ${ }^{13,14}$.

Nesse sentido, o termo bareback vem se tornando comum em MSE, deixando de ser uma das categorias para o público gay e passando a ser a norma. Porém, o aumento dessa prática pode estar contribuindo para um incremento na prevalência de HIV e outras infecções sexualmente transmissíveis (IST) em HSH ${ }^{15,16}$.

Com relação ao Brasil, de fato, o consumo de MSE é expressivo no país. Um relatório do site Pornhub, uma das maiores plataformas de vídeos eróticos do mundo, contabilizou 81 milhões de visitantes por dia, com 28,5 bilhões de visitantes ao ano. O Brasil ficou em décimo lugar entre os países que mais acessaram o Pornhub, sendo predominante a busca por pornografia gay ${ }^{17}$. Paralelo a isso, foi o único país latino-americano a ter aumento de novas infecções no último relatório da UNAIDS, sendo também o território com o maior número de pessoas vivendo com o HIV/ Aids na região da América Latina ${ }^{18}$.

Uma vez que não há estudos que avaliem esse objeto no país, visamos avaliar o consumo de MSE na modalidade bareback por homens que fazem sexo com homens no Brasil e a sua associação com a prática de sexo anal sem preservativo.

\section{Materiais e métodos}

Trata-se de um estudo observacional, transversal, analítico, com utilização de inquérito on-line de abrangência nacional (Brasil), realizado com HSH. Empregou-se amostra intencional com base na população de $3,5 \%$ de HSH conforme o Ministério da Saúde do Brasil recomenda ${ }^{19}$. O cálculo do tamanho da amostra levou ainda em consideração um erro máximo tolerável de 5\% e um nível de significância de 5\%, sendo a amostra final fixada em 2.248 participantes. Os critérios de elegibilidade foram residir em território brasileiro, identificar-se como homem cisgênero, ter 
18 anos ou mais e ter tido ao menos uma relação sexual com outro homem nos últimos 12 meses.

Para a obtenção dos dados, uma página foi criada no Facebook ${ }^{\circledR}$ (https://www.facebook. com/taafimdeque/) com uma postagem fixa que continha detalhes da pesquisa e convite para participação. Essa postagem foi impulsionada de modo a atingir participantes on-line em todas as regiões do Brasil. Concomitantemente houve a divulgação da pesquisa em grupos do Facebook ${ }^{\circledR}$ voltados à temática bareback ou de MSE. O usuário tinha acesso a um link que o direcionava para o questionário do estudo hospedado no Google Forms. Foram incluídos os internautas que atenderam os critérios de seleção, assinalaram on-line o Termo de Consentimento Livre e Esclarecido e preencheram todos os itens do questionário. Os participantes deveriam informar o e-mail para evitar duplicidade de resposta.

O questionário de pesquisa foi subdividido em quatro seções: (i) características pessoais; (ii) características socioculturais; (iii) questões de saúde; e (iv) consumo de mídia sexualmente explícita. Sobre o consumo de MSE, os participantes foram perguntados: a) se preferem filmes com cenas com preservativo ou sem; b) a idade em que começaram a ver pornografia; c) quantas cenas costumam ver por semana (considerando que uma cena contenha em média 20 minutos); d) quantos minutos de pornografia bareback assistem por semana (considerando que uma cena contenha em média 20 minutos); e) a principal forma de acesso a pornografia; f) se, na sua percepção, o consumo de pornografia bareback altera suas práticas sexuais; g) se considera o sexo bareback um fetiche; e h) se considera o sexo bareback um fetiche realizável, ou seja, que pode ser realizado na prática. Questões relacionadas a posicionamento sexual (insertivo/receptivo/versátil) e sexo anal sem preservativo (30 dias a 6 meses anteriores à pesquisa) também foram levantadas por questões diretas com desfecho binário (sim ou não). A coleta ocorreu em setembro de 2017.

A análise descritiva das variáveis numéricas e categóricas foi realizada com auxílio do Software Statistical Package for the Social Science (SPSS) $\mathrm{IBM}^{\circledR}$ versão 26.0. Para análise bivariada das variáveis de interesse em relação à preferência por MSE na modalidade bareback, utilizaram-se os testes de qui-quadrado ou Exato de Fisher, sendo que aquelas variáveis que apresentaram $\mathrm{p}<0,50$ foram levadas para o modelo de regressão logística multivariada. As odds ratio (OR) brutas e ajustadas (ORa) foram obtidas com vista a avaliar fatores relacionados à chance de envolvi- mento em sexo anal desprotegido, adotando-se o desfecho binário "envolveu-se em sexo anal sem preservativo? (sim, não)”. As variáveis com valor de $\mathrm{p}<0,20$ foram admitidas para a construção do modelo de regressão logística ajustado com método de entrada forward conditional. O nível de significância adotado foi de 5\% e intervalos de confiança de $95 \%$. Foi considerada a melhor performance do modelo multivariado com aspectos de acurácia, sensibilidade e especificidade (Receiver Operating Characteristic - ROC), provando que o desempenho estatístico desenvolvido foi melhor que o acaso.

$\mathrm{O}$ estudo atendeu às normas nacionais $\mathrm{e}$ internacionais de ética em pesquisa com seres humanos e foi aprovado pelo Comitê de Ética em Pesquisa da Universidade Federal do Piauí, Brasil.

\section{Resultados}

Participaram do estudo 2.248 HSH, com média de idade de 24,4 anos (DP $\pm 5,6)$. O mais frequente entre os HSH estudados era ser solteiro $(69,1 \%)$, possuir ensino superior $(81,9 \%)$, ter parceria sexual casual $(68,9 \%)$, identificar-se como homossexual $(85,3 \%)$ e ter status sorológico negativo para o HIV (49,1\%). Em média, os participantes tiveram 3,9 parceiros nos últimos 30 dias, e um percentual considerável $(33,3 \%)$ teve mais de três parceiros (Tabela 1).

Na Tabela 2 apresentamos os resultados da associação entre as características sociodemográficas e comportamentais com o desfecho sexo sem preservativo ao menos uma vez nos seis meses anteriores à coleta de dados. À exceção do status sorológico para o HIV $(0,103)$, todas as variáveis foram estatisticamente significativas.

Já a Tabela 3 apresenta a associação entre as práticas de sexo sem preservativo e a preferência pelo consumo de cenas na categoria bareback, em que todas as variáveis foram estatisticamente significantes.

Para avaliar a influência das características sociais, demográficas, comportamentais e de consumo no sexo anal desprotegido, optamos pelo modelo de regressão logística, através do qual se observou que aumentaram as chances de se envolver em sexo anal sem uso de preservativo: (i) ter tido três ou mais parceiros nos últimos 30 dias (ORa=9,4 vezes); (ii) ter a prática bareback como fetiche realizável $(\mathrm{ORa}=3,5)$; (iii) preferir MSE com cenas bareback $(\mathrm{ORa}=2,6)$; (iv) ter parceria sexual casual $(\mathrm{ORa}=1,8)$; e $(\mathrm{v})$ possuir conhecimento sobre o status sorológico negati- 
Tabela 1. Características sociodemográficas, sexuais e sorológicas dos homens que fazem sexo com homens e seus parceiros sexuais $(n=2.248)$.

\begin{tabular}{|c|c|c|}
\hline Variável & $\mathbf{n}$ & $\%$ \\
\hline \multicolumn{3}{|l|}{ Idade em anos } \\
\hline $18-20$ & 530 & 23,6 \\
\hline $21-30$ & 1.466 & 65,2 \\
\hline$>30$ & 252 & 11,2 \\
\hline \multicolumn{3}{|l|}{ Escolaridade } \\
\hline Ensino médio ou inferior & 112 & 5,0 \\
\hline Ensino superior & 1.841 & 81,9 \\
\hline Pós-Graduação & 295 & 13,1 \\
\hline \multicolumn{3}{|l|}{ Estado civil } \\
\hline Solteiro & 1.554 & 69,1 \\
\hline Em relacionamento & 694 & 30,9 \\
\hline \multicolumn{3}{|l|}{ Orientação sexual } \\
\hline Homossexual & 1.917 & 85,3 \\
\hline Bissexual & 265 & 11,8 \\
\hline Heterossexual & 26 & 1,1 \\
\hline Pansexual & 40 & 1,8 \\
\hline \multicolumn{3}{|l|}{ Tipo de parcerias estabelecidas } \\
\hline Casual & 1.043 & 46,4 \\
\hline Fixo & 699 & 31,1 \\
\hline Fixo e casual & 506 & 22,5 \\
\hline \multicolumn{3}{|c|}{ Status sorológico do último parceiro } \\
\hline $\mathrm{HIV}+$ & 72 & 3,2 \\
\hline HIV- & 1.104 & 49,1 \\
\hline Não sei & 1.072 & 47,7 \\
\hline \multicolumn{3}{|c|}{ Parceiros sexuais nos últimos 30 dias } \\
\hline Não teve & 293 & 13,0 \\
\hline 1 ou 2 & 1.207 & 53,7 \\
\hline 3 ou mais & 748 & 33,3 \\
\hline
\end{tabular}

vo do parceiro para o HIV $(\mathrm{ORa}=1,4)$. Em contrapartida, constituíram fatores de proteção: (i) não ter a prática bareback como fetiche; (ii) estar em um relacionamento; e (iii) relacionar-se com parceiro soropositivo (Tabela 4).

\section{Discussão}

Neste estudo de abrangência nacional, registramos associação entre preferir assistir a MSE na modalidade bareback e práticas de sexo anal sem preservativo entre HSH brasileiros. A quantidade de parceiros, o tipo de parceria sexual estabelecida, as estratégias de proteção adotadas e a fetichização da prática bareback parecem aumentar consideravelmente as chances de o indivíduo se envolver em sexo anal sem preservativo.
A preferência por filmes na categoria bareba$c k$ esteve significativamente associada à prática de sexo sem camisinha entre os participantes, com sexo anal insertivo, sexo anal receptivo e sexo oral, aumentando em 2,6 vezes as chances dos HSH de se envolverem em sexo anal sem camisinha. Tal dado é similar ao que já foi descrito por outros autores nos Estados Unidos ${ }^{6}$, na Noruega $^{20}$ e na Austrália ${ }^{21}$, corroborando a existência de uma ligação direta entre a preferência por cenas bareback e as intenções de sexo sem preservativo.

De acordo com a literatura, os HSH em relacionamento com parceiro fixo tendem a consumir mais MSE bareback e realizar mais sexo sem camisinha do que indivíduos solteiros, que possuem múltiplas parcerias e que fazem sexo casual $^{22}$. Tal comportamento pode ser explicado porque no começo do relacionamento os casais são mais inseguros e propensos a se proteger. Entretanto, à medida que este se torna duradouro, a confiança e a familiaridade com o parceiro crescem e a percepção de risco diminui, fazendo com que os envolvidos diminuam a frequência de uso ou passem a não utilizar preservativo em suas relações sexuais com parceiro fixo ${ }^{23}$.

No entanto, pôde-se observar que a visualização de MSE bareback pode contribuir significativamente para aumento na concretização de sexo anal sem preservativo não somente entre aqueles que mantêm uma relação estável, mas também entre os que praticam sexo casual e com múltiplos parceiros, em especial os mais jovens ${ }^{24,25}$.

Nossos achados demonstram que quase metade dos participantes relatou ter conhecimento sobre o status sorológico negativo do último parceiro para o HIV, e esse fato foi associado a maiores chances de envolvimento em sexo anal sem preservativo. Isso pode ser decorrente do atual serosorting, termo utilizado para denominar a escolha de parceiros sexuais com base no status para $\mathrm{HIV}^{26}$. Estudos apontam que essa prática tem sido bastante utilizada por HSH como um determinante para a realização de sexo sem preservativo entre indivíduos soroconcordantes, tanto em soronegativos quanto em soropositivos, sobretudo quando há familiaridade entre os parceiros ${ }^{27,28}$.

Entre os participantes da pesquisa, $47,7 \%$ relataram não ter ciência do status do último parceiro. Desses, apenas um terço referiu sexo bareback nos últimos 30 dias, reforçando que o desconhecimento do status do parceiro pode fazer com que esses indivíduos prefiram o sexo com preservativo ${ }^{27}$.

No entanto, há evidências de que o aumento do serosorting relacionado ao sexo anal sem 
Tabela 2. Análise bivariada da associação entre características sociodemográficas e comportamentais com práticas de sexo sem preservativo $(\mathrm{n}=2.248)$.

\begin{tabular}{|c|c|c|c|c|c|c|c|}
\hline \multirow{3}{*}{ Variáveis } & \multicolumn{6}{|c|}{ Sexo sem preservativo (últimos 6 meses) } & \multirow{3}{*}{ p-valor } \\
\hline & \multicolumn{2}{|c|}{ Sim } & \multicolumn{2}{|c|}{ Não } & \multicolumn{2}{|c|}{ Total } & \\
\hline & $\mathbf{n}$ & $\%$ & $\mathbf{n}$ & $\%$ & $\mathbf{n}$ & $\%$ & \\
\hline \multicolumn{8}{|l|}{ Idade } \\
\hline $18-20$ & 203 & 38,3 & 327 & 61,7 & 530 & 23,6 & $<0,001$ \\
\hline $21-30$ & 763 & 52 & 703 & 48 & 1.466 & 65,2 & \\
\hline$>30$ & 143 & 56,7 & 109 & 43,3 & 252 & 11,2 & \\
\hline \multicolumn{8}{|l|}{ Escolaridade } \\
\hline Ensino médio ou inferior & 48 & 42,9 & 64 & 57,1 & 112 & 5,0 & 0,045 \\
\hline Ensino superior & 895 & 48,6 & 946 & 51,4 & 1.841 & 81,9 & \\
\hline Pós-Graduação & 166 & 56,3 & 129 & 43,7 & 295 & 13,1 & \\
\hline \multicolumn{8}{|l|}{ Estado civil } \\
\hline Solteiro & 629 & 40,5 & 925 & 59,5 & 1.554 & 69,1 & $<0,001$ \\
\hline Em relacionamento & 480 & 69,2 & 214 & 30,8 & 694 & 30,9 & \\
\hline \multicolumn{8}{|l|}{ Orientação sexual } \\
\hline Homossexual & 977 & 51,0 & 940 & 49,0 & 1.917 & 85,3 & $<0,001$ \\
\hline Bissexual & 118 & 44,5 & 147 & 55,5 & 265 & 11,8 & \\
\hline Heterossexual & 10 & 38,5 & 16 & 61,5 & 26 & 1,2 & \\
\hline Pansexual & 4 & 10,0 & 36 & 90,0 & 40 & 1,8 & \\
\hline \multicolumn{8}{|c|}{ Status sorológico para o HIV (autorrelato) } \\
\hline $\mathrm{HIV}+$ & 77 & 58,3 & 55 & 41,7 & 132 & 5,9 & 0,103 \\
\hline HIV- & 881 & 48,8 & 925 & 51,2 & 1.806 & 80,3 & \\
\hline Não sei & 151 & 48,7 & 159 & 51,3 & 310 & 13,8 & \\
\hline \multicolumn{8}{|c|}{ Tipo de parcerias sexuais estabelecidas } \\
\hline Casual & 389 & 37,3 & 654 & 62,7 & 1.043 & 46,4 & $<0,001$ \\
\hline Fixo & 455 & 65,1 & 244 & 34,9 & 699 & 31,1 & \\
\hline Fixo e casual & 265 & 52,4 & 241 & 47,6 & 506 & 22,5 & \\
\hline \multicolumn{8}{|c|}{ Número de parceiros nos últimos 30 dias } \\
\hline Não teve & 19 & 6,5 & 274 & 93,5 & 293 & 13,0 & $<0,001$ \\
\hline Entre 1 e 2 & 631 & 52,3 & 576 & 47,7 & 1.207 & 53,7 & \\
\hline$\geq 3$ & 459 & 61,4 & 289 & 38,6 & 748 & 33,3 & \\
\hline \multicolumn{8}{|c|}{ Status sorológico para HIV do último parceiro } \\
\hline $\mathrm{HIV}+$ & 34 & 47,2 & 38 & 52,8 & 72 & 3,2 & $<0,001$ \\
\hline HIV- & 689 & 62,4 & 415 & 37,6 & 1.104 & 49,1 & \\
\hline Não sei & 386 & 36 & 686 & 64 & 1.072 & 47,7 & \\
\hline
\end{tabular}

Fonte: Elaborado pelos autores.

preservativo em $\mathrm{HSH}$ pode contribuir para o aumento na incidência de outras IST, como sífilis $^{29}$, clamídia, gonorreia ${ }^{30}$ e hepatite $\mathrm{C}^{31}$, além do próprio HIV, considerando-se a existência dos períodos de janela imunológica e os riscos associados. As MSE desempenham papel importante nesse processo, pois homens sabidamente soronegativos que consomem MSE bareback são mais tentados a realizá-lo do que os que consomem MSE com relação protegida ${ }^{32}$.

Essa conexão fundamenta-se no fato de o comportamento humano possuir uma base potencial para imitação, ou seja, os indivíduos, cons- ciente ou inconscientemente, tendem a reproduzir atitudes realizadas pelos outros, influenciadas por aspectos sociais, cognitivos, emocionais, que traduzam direta ou indiretamente suas preferências, mesmo que representem algum risco à saú$\mathrm{de}^{33,34}$. Desse modo, a exposição às MSE bareback pode despertar o interesse no espectador a longo ou curto prazo, fazendo com que este dirija o seu desejo/fantasia para essa prática, tornando-a um fetiche e fomentando a curiosidade em realizá-lo.

Curiosamente, verificamos que o número de participantes que consideram o bareback um fetiche foi semelhante entre os que o praticam e 
Tabela 3. Análise bivariada da associação entre preferir assistir a filme na categoria bareback e práticas de sexo sem preservativo $(n=2.248)$.

\begin{tabular}{|c|c|c|c|c|c|c|c|}
\hline \multirow{3}{*}{ Variáveis } & \multicolumn{6}{|c|}{ Preferência por assistir a filmes bareback } & \multirow{3}{*}{ p-valor } \\
\hline & \multicolumn{2}{|c|}{$\operatorname{Sim}(n=982)$} & \multicolumn{2}{|c|}{ Não $(n=1.266)$} & \multicolumn{2}{|c|}{ Total $(\mathrm{n}=2.248)$} & \\
\hline & $\mathbf{n}$ & $\%$ & $\mathbf{n}$ & $\%$ & $\mathbf{n}$ & $\%$ & \\
\hline \multicolumn{8}{|c|}{$\begin{array}{l}\text { Envolveu-se em sexo bareback (insertivo ou } \\
\text { receptivo) nos últimos } 6 \text { meses? }\end{array}$} \\
\hline Sim & 568 & 25,3 & 541 & 24,0 & 1.109 & 49,3 & $<0,001$ \\
\hline Não & 414 & 18,4 & 725 & 32,3 & 1.139 & 50,7 & \\
\hline \multicolumn{8}{|c|}{$\begin{array}{l}\text { Sexo bareback como insertivo sem uso de } \\
\text { preservativo nos últimos } 30 \text { dias? }\end{array}$} \\
\hline Sim & 367 & 16,3 & 375 & 16,7 & 742 & 33,0 & $<0,001$ \\
\hline Não & 615 & 27,4 & 891 & 39,6 & 1.506 & 67,0 & \\
\hline \multicolumn{8}{|c|}{$\begin{array}{l}\text { Sexo bareback como receptivo sem uso de } \\
\text { preservativo nos últimos } 30 \text { dias? }\end{array}$} \\
\hline Sim & 402 & 17,9 & 370 & 16,5 & 772 & 34,4 & $<0,001$ \\
\hline Não & 580 & 25,8 & 896 & 39,8 & 1.476 & 65,6 & \\
\hline \multicolumn{8}{|c|}{$\begin{array}{l}\text { Sexo oral sem uso de preservativo nos } \\
\text { últimos } 30 \text { dias? }\end{array}$} \\
\hline $\operatorname{Sim}$ & 803 & 35,7 & 974 & 43,3 & 1.777 & 79,0 & 0,006 \\
\hline Não & 179 & 8,0 & 292 & 13,0 & 471 & 21,0 & \\
\hline \multicolumn{8}{|c|}{ Considera o bareback como fetiche? } \\
\hline Sim & 697 & 31,0 & 474 & 21,1 & 1.171 & 52,1 & $<0,001$ \\
\hline Não & 285 & 12,7 & 792 & 35,2 & 1.077 & 47,9 & \\
\hline \multicolumn{8}{|c|}{$\begin{array}{l}\text { Considera bareback como um fetiche } \\
\text { realizável? }\end{array}$} \\
\hline Sim & 410 & 69,6 & 287 & 49,3 & 589 & 50,3 & $<0,001$ \\
\hline Não & 179 & 30,4 & 295 & 50,7 & 582 & 49,7 & \\
\hline \multicolumn{8}{|c|}{$\begin{array}{l}\text { Acredita que ver filme bareback influencia } \\
\text { na prática sexual? }\end{array}$} \\
\hline Sim & 499 & 22,2 & 580 & 25,8 & 1.079 & 48,0 & 0,019 \\
\hline Não & 483 & 21,5 & 686 & 30,5 & 1.169 & 52,0 & \\
\hline
\end{tabular}

Fonte: Elaborado pelos autores.

os que não o praticam. Esse resultado corrobora pesquisas anteriores ${ }^{20,35}$, sugerindo que ter a prática bareback como fetiche pode representar um fator positivo quando percebido como uma substituição da realidade (sexo anal real), ou seja, possuem essa prática como fetiche, mas não o realizam - então ficam apenas o desejando.

Por outro lado, o fetiche pode levar ao consumo excessivo e problemático dessas mídias, possibilitando a tendência de enxergar esse comportamento como um estímulo à prática na busca por novas sensações, o que aumenta as chances de realização de sexo anal sem preservativo ${ }^{16,24}$.

Portanto, são necessárias mais pesquisas que melhor investiguem se os HSH preferem as MSE bareback porque eles já praticam esse tipo de sexo, ou se essa predileção corresponde a um desejo ou uma fantasia interna, mas não representa seus comportamentos sexuais. Assim como é preciso repensar as estratégias de prevenção para essa população, com ênfase em medidas que abordem a erotização do sexo seguro e o esclarecimento de crenças sexuais preexistentes, especialmente entre os mais jovens, e que contemplem as novas formas de prevenção do HIV e fortaleçam a importância do preservativo na prevenção de outras IST.

Este estudo possui algumas limitações a serem apontadas. Primeiro, o fato de as informações terem sido autorrelatadas e serem passíveis de vieses de memória e desejabilidade social. Embora possa haver ressalvas sobre os dados do ponto de vista da precisão das informações, a literatura é repleta de estudos que reforçam a viabilidade de se estudar assuntos que envolvem tabus e agravos carregados de estigma e precon- 
Tabela 4. Análise multivariada de fatores associados a envolver-se em sexo anal sem preservativo.

\begin{tabular}{|c|c|c|c|}
\hline Variável & ORa & IC95\% & p-valor \\
\hline \multicolumn{4}{|l|}{ Relacionamento } \\
\hline Não estar em um relacionamento & 1 & & \\
\hline Estar em um relacionamento & 0,6 & $0,3-0,9$ & 0,48 \\
\hline Não conhecer o status sorológico do parceiro & 1 & & \\
\hline Parceiro soropositivo & 0,7 & $0,4-1,0$ & 0,034 \\
\hline Status negativo & 1,4 & $1,1-2,3$ & 0,058 \\
\hline \multicolumn{4}{|l|}{ Quantidade de parceiros sexuais nos últimos 30 dias } \\
\hline Nenhum & 1 & & \\
\hline $1-2$ & 1,8 & $1,2-2,8$ & 0,004 \\
\hline$\geq 3$ & 9,4 & $3,9-22,4$ & $<0,001$ \\
\hline \multicolumn{4}{|l|}{ Tipo de parcerias sexuais estabelecidas } \\
\hline Parceiro fixo & 1 & & \\
\hline Parceiro casual & 1,8 & $1,5-1,9$ & $<0,001$ \\
\hline Parceiro fixo e casual & 0,91 & $0,54-1,5$ & 0,721 \\
\hline \multicolumn{4}{|l|}{ Consumo de mídia sexualmente explícita tipo bareback } \\
\hline Não consome & 1 & & \\
\hline Consome & 1,4 & $1,2-1,7$ & $<0,001$ \\
\hline \multicolumn{4}{|l|}{ Preferência por cenas } \\
\hline Com preservativo & 1 & & \\
\hline Com sexo bareback & 2,6 & $1,5-4,6$ & 0,001 \\
\hline Sem preferência & 1,7 & $1,4-2,1$ & $<0,001$ \\
\hline \multicolumn{4}{|l|}{ Bareback como fetiche } \\
\hline Sim & 1 & & \\
\hline Não & 0,49 & $0,32-0,76$ & 0,001 \\
\hline \multicolumn{4}{|l|}{ Bareback como fetiche realizável } \\
\hline Não & 1 & & \\
\hline Sim & 3,52 & $2,3-5,4$ & $<0,001$ \\
\hline
\end{tabular}

ORa: odds ratios ajustada; IC: intervalo de confiança.

Fonte: Elaborado pelos autores.

ceito, como HIV/Aids, principalmente em populações de difícil acesso, através de dados autorrelatados ${ }^{36,37}$. Segundo, destacamos que respostas incompletas não foram salvas no Google Forms. Terceiro, ressaltamos que a divulgação em grupos específicos na rede social Facebook ${ }^{\circledR}$, voltados para o tema de práticas sexuais entre $\mathrm{HSH}$, pode ter implicado a ocorrência de viés de seleção da amostra e influenciado nos resultados.

Destacamos também que, embora a pesquisa tenha sido realizada em todos os estados das cinco regiões brasileiras, a ausência de cálculo amostral dificulta a generalização dos dados. Por fim, a pesquisa foi realizada quando não estava disponível gratuitamente no Brasil a Profilaxia Pré-exposição (PrEP), o que poderia fornecer achados interessantes relacionados ao gerenciamento do sexo anal sem preservativo.

\section{Conclusão}

Verificamos associação entre o consumo de MSE na modalidade bareback e a prática de sexo sem preservativo entre $\mathrm{HSH}$, em que a preferência por mídias que retratam esse tipo de sexo, a ideia de considerá-lo um fetiche, o tipo de parceria sexual e o conhecimento do status sorológico do parceiro podem aumentar as chances de envolvimento em sexo anal sem preservativo.

Este estudo, assim como grande parte das pesquisas já realizadas sobre a influência de MSE sobre o comportamento sexual de HSH, considerou apenas a prática de sexo sem preservativo, excluindo-se a PrEP e outras formas de prevenção combinada. Dessa forma, sugere-se ainda o desenvolvimento de estudos posteriores que avaliem a relação entre uso das novas formas de prevenção, o consumo de MSE e o comportamento sexual de $\mathrm{HSH}$. 


\section{Colaboradores}

AFL Sousa, AAFLN Queiroz e IAC Mendes: concepção, coleta, planejamento, análise, interpretação e redação do trabalho. AA Martins, OP Frota, TME Araújo e I Fronteira: análise, interpretação e redação do trabalho. Todos os autores aprovaram a versão final a ser publicada.

\section{Referências}

1. Hald GM. Gender differences in pornography consumption among young heterosexual Danish adults. Arch Sex Behav 2006;35(5):577-585.

2. Downing MJ Jr, Schrimshaw EW, Scheinmann R, Antebi-Gruszka N, Hirshfield S. Sexually Explicit Media Use by Sexual Identity: A Comparative Analysis of Gay, Bisexual, and Heterosexual Men in the United States. Arch Sex Behav 2017; 46(6):1763-1776.

3. Giano Z. The influence of online experiences: the shaping of gay male identities. J Homosex 2019; 18:1-15.

4. Nelson KM, Perry NS, Carey MP. Sexually explicit media use among 14-17-year-old sexual minority males in the U.S. Arch Sex Behav 2019; 48(8):2345-2355.

5. Hald GM, Smolenski D, Rosser BR. Perceived effects of sexually explicit media among men who have sex with men and psychometric properties of the Pornography Consumption Effects Scale (PCES). J Sex Med 2013; 10(3):757-767.

6. Schirmshaw EW, Antebi-Gruszka N, Downing Jr MJ. Viewing of Internet-based sexually explicit media as a risk factor for condomless anal sex among men who have sex with men in four U.S. cities. Plos ONE 2016; 11(4):1-11

7. Carballo-Diéguez A, Ventuneac A, Bauermeister J, Dowsett GW, Dolezal C, Remien RH, Balan I, Rowe M. Is 'bareback' a useful construct in primary HIV -prevention? Definitions, identity and research. Cult Health Sex 2009; 11(1):51-65.

8. Halkitis PN, Parsons JT, Wilton L. Barebacking among gay and bisexual men in New York City: Explanations for the emergence of intentional unsafe behavior. Arch Sex Behav 2003; 32(4):351-357.

9. Halkitis PN, Siconolfi D, Fumerton M, Barlup K. Facilitators of barebacking among emergent adult gay and bisexual men: implications for HIV prevention. J LGBT Health Res 2008; 4(1):11-26.

10. Brennan J. Gay Porn's Bareback Momentum. J Homosex 2020; 67(1):127-157.

11. Mowlabocus S, Harbottle J. Witzel C. What we can't see? Understanding the representations and meanings of UAI, barebacking, and semen exchange in gay male pornography. J Homosex 2014; 61(10):1462-1480.

12. Terrell M. Gay Porn Bareback: Skyn Tight [Internet]. 2019 [acessado 13 out 2018]. Disponível em: https:// slate.com/human-interest/2019/09/gay-porn-bareback-magic-condom-trick.html.

13. Dean T. Mediated intimacies: raw sex, Truvada and the biopolitcs of chemoprophylaxis. Sexualites 2015; 18(1):224-246.

14. Tollini C. How to holdouts went bareback: Cocky Boys and Men.com's initial transition to producing videos without condoms. Porn Studies 2019; 6(3):282-300.

15. Rosser BR, Grey JA, Wilkerson JM, Iantaffi A, Brady SS, Smolenski DJ, Horvath KJ. A commentary on the role of sexually explicit media (SEM) in the transmission and prevention of HIV among men who have sex with men (MSM). AIDS Behav 2012; 16(6):13731381.

16. Rosser BR, Smolenski DJ, Erickson D, Iantaffi A, Brady SS, Grey JA, Hald GM, Horvath KJ, Kilian G, Træen B, Wilkerson JM. The effects of gay sexually explicit media on the HIV risk behavior of men who have sex with men. AIDS Behav 2013; 17(4):1488-1498. 
17. CANALTECH. Pornhub divulga estatísticas de 2017 e mostra que brasileiro adora pornografia [Internet]. 2018 [acessado 13 out 2018]. Disponível em: https:// canaltech.com.br/comportamento/pornhub-divulga -estatisticas-de-2017-e-mostra-que-brasileiro-adora -pornografia-106304/.

18. Joint United Nations Programme on HIV/AIDS (UNAIDS). Global Report UNAIDS DATA 2019. Geneva: UNAIDS; 2019.

19. Brasil. Ministério da Saúde (MS). Secretaria de Vigilância em Saúde. Pesquisa de conhecimento, atitudes e práticas na população brasileira. Brasília: MS; 2011.

20. Træen B, Noor SW, Hald GM, Rosser BR, Brady SS, Erickson D, Galos DL, Grey JA, Horvath KJ, Iantaffi A, Kilian G, Wilkerson JM. Examining the relationship between use of sexually explicit media and sexual risk behavior in a sample of men who have sex with men in Norway. Scand J Psychol 2015; 56(3):290-296.

21. Thai M, Barlow FK. Bareback sexually explicit media consumption and men who have sex with men's responses to sexual partners who prefer anal intercourse with or without condoms. Arch Sex Behav 2019; 48(4):1191-1201.

22. Newcomb ME, Ryan DT, Garofalo R, Mustanski B. The effects of sexual partnership and relationship characteristics on three sexual risk variables in young men who have sex with men. Arch Sex Behav 2014; 43(1):61-72.

23. Xu Y, Zheng Y, Rahman Q. The relationship between self-reported sexually explicit media consumption and sexual risk behaviors among men who have sex with men in China. J Sex Med 2017; 14(3):357-365.

24. Whitfield THF, Rendina HJ, Grov C, Parsons JT. Sexually Explicit Media and Condomless Anal Sex Among Gay and Bisexual Men. AIDS Behav 2018; 22(2):681689.

25. Weinberg MS, Williams CJ, Kleiner S, Irizarry Y. Pornography, normalization, and empowerment. Arch Sex Behav 2010; 39(6):1389-1401.

26. Wilson DP, Regan DG, Heymer KJ, Jin F, Prestage GP, Grulich AE. Serosorting may increase the risk of HIV acquisition among men who have sex with men. Sex Transm Dis 2010; 37(1):13-17.

27. Matser A, Heijman T, Geskus R, de Vries H, Kretzschmar M, Speksnijder A, Xiridou M, Fennema H, van der Loeff MS. Perceived HIV status is key determinant of unprotected anal intercourse within partnerships of men who have sex with men in Amsterdam. AIDS Behav 2014; 18(12):2442-2456.

28. Grewal R, Allen VG, Gardner S, Moravan V, Tan DH, Raboud J, Bayoumi AM, Kaul R, Mazzulli T, McGee F, Rourke SB, Burchell AN, OHTN Cohort Study Research Team. Serosorting and recreational drug use are risk factors for diagnosis of genital infection with chlamydia and gonorrhoea among HIV-positive men who have sex with men: results from a clinical cohort in Ontario, Canada. Sex Transm Infect 2017; 93(1):7175 .
29. Grov C, Jonathan Rendina H, Patel VV, Kelvin E, Anastos K, Parsons JT. Prevalence of and Factors Associated with the Use of HIV Serosorting and Other Biomedical Prevention Strategies Among Men Who Have Sex with Men in a US Nationwide Survey. AIDS Behav 2018; 22(8):2743-2755.

30. Khosropour CM, Dombrowski JC, Swanson F, Kerani RP, Katz DA, Barbee LA, Hughes JP, Manhart LE, Golden MR. Trends in Serosorting and the Association With HIV/STI Risk Over Time Among Men Who Have Sex With Men. J Acquir Immune Defic Syndr 2016; 72(2):189-197.

31. Apers L, Vanden Berghe W, De Wit S, Kabeya K, Callens S, Buyze J, Kenyon C, Florence E, Buvé A. Risk factors of HCV acquisition among HIV-positive MSM in Belgium. J Acquir Immune Defic Syndr 2015; 68(5):585-593.

32. Jonas KJ, Hawk ST, Vastenburg D, de Groot P. "Bareback" pornography consumption and safe-sex intentions of men having sex with men. Arch Sex Behav 2014; 43(4):745-753.

33. Bandura A. Self-efficacy: Toward a unifying theory of behavioral change. Psych Rev 1977; 84(2):191-215.

34. Chartrand TL, Lakin JL. The antecedents and consequences of human behavioral mimicry. Annu Rev Psychol 2013; 64:285-308.

35. Nelson KM, Eaton LA, Gamarel KE. Preferences for Condomless Sex in Sexually Explicit Media Among Black/African American Men Who Have Sex with Men: Implications for HIV Prevention. Arch Sex Behav 2017; 46(4):977-985.

36. Queiroz AAFLN, Sousa AFL, Brignol S, Araújo TME, Reis RK. Vulnerability to HIV among older men who have sex with men users of dating apps in Brazil. Braz J Infect Dis 2019; 23(5):298-306.

37. Queiroz AAFLN, Sousa ÁFL, Matos MCB, Araújo TME, Brignol S, Reis RK, Gir E, Moura MEB. Factors associated with self-reported non-completion of the hepatitis $B$ vaccine series in men who have sex with men in Brazil. BMC Infect Dis 2019; 19(1):335.

Artigo apresentado em 06/05/2020

Aprovado em 04/10/2020

Versão final apresentada em 06/10/2020

Editores-chefes: Romeu Gomes, Antônio Augusto Moura da Silva 
